\title{
Advances in technology and business potential of new drug delivery systems
}

\author{
Munira Momin ${ }^{1} \cdot$ John Disouza $^{2} \cdot$ Vandana Patravale $^{3}$
}

Published online: 30 June 2016

(C) Controlled Release Society 2016

The pharmaceutical business is growing at a phenomenal rate with novel drug delivery systems being the cardinal driver for the conspicuous upsurge. Novel drug delivery systems offer superior therapeutic potential by addressing market and/or patient needs and offer patient compliance through enhanced efficacy, reduced dose/dosing frequency, decreased toxicity, and ease of administration. In view of these advantages, the growth of these innovative drug delivery systems is also boosted by fast track approvals from regulatory authorities to ensure their rapid entry into the market.

This special issue of Drug Delivery and Translational Research has materialized from the 14th International symposium of Controlled Release Society-Indian Chapter on "Advances in Technology and Business Potential of New Drug Delivery Systems" organized at the Institute of Chemical Technology, Mumbai, India. The issue encompasses an eclectic blend of review and research papers pertaining to different aspects of novel drug delivery.

The issue features three review articles which focus on targeted nanosized drug delivery systems for antimalarial combination therapy as an upcoming strategy to tackle the peril of malaria; challenges and potential strategies in traversing the transungual barrier for nail infections and future avenues in

Vandana Patravale

vb.patravale@ictmumbai.edu.in

1 SVKM's Dr. Bhanuben Nanavati College of Pharmacy, SVKM Mumbai Campus, Mumbai, Maharashtra, India

2 Tatyasaheb Kore College of Pharmacy, Warananagar, Maharashtra, India

3 Department of Pharmaceutical Sciences and Technology, Institute of Chemical Technology, Mumbai, Maharashtra, India exploring this route for systemic drug therapy; and nanocrystals for ocular delivery as a promising approach for enhanced activity where there is a limitation on use of novel drug delivery systems with high surfactant concentration. Additionally, a brief communication on the novel bottom-up spray drying based technology (NanoCrySP) for the generation of nanocrystals is also published.

The issue includes three research papers dealing with the use of solid dispersions to enhance dissolution and in turn bioavailability of poorly soluble drug; use of solid lipid nanoparticles of amphotericin B to achieve bioenhancement with reduce toxicity exhibiting a strong potential to treat systemic fungal infection; and studies on chitosan-based mucoadhesive systems for mucosal protein delivery addressing the challenge in transport of macromolecules with enhanced bioavailability.

To summarize, this issue gives an insight into the upcoming strategies in novel drug delivery science which in our vision holds strong business opportunities that will drive the pharmaceutical business in recent years.

\section{Compliance with ethical standards}

Conflict of interest The authors declare that they have no conflict of interest. 